\title{
Moving Coalition Analysis for Collaboration Evaluation in Overall Equipment Effectiveness
}

\author{
M. A. Mansor ${ }^{1}$ and A. Ohsato ${ }^{2}$ \\ ${ }^{1}$ Faculty of Mechanical Engineering, Universiti Malaysia Pahang, Lebuhraya Tun Razak, 26300 UMP Kuantan, Pahang, Malaysia \\ ${ }^{2}$ Department of Information Science and Control Engineering, Nagaoka University of Technology, Kamitomioka 1603-1, \\ Nagaoka 940-2188, Japan
}

Correspondence should be addressed to M. A. Mansor, ariffinmansor@yahoo.com

Received 21 November 2009; Revised 7 January 2010; Accepted 21 January 2010

Copyright (C) 2010 M. A. Mansor and A. Ohsato. This is an open access article distributed under the Creative Commons Attribution License, which permits unrestricted use, distribution, and reproduction in any medium, provided the original work is properly cited.

\begin{abstract}
This paper proposed a method to evaluate collaboration of criteria such as Performance Rate, Quality Rate, and Availability of Overall Equipment Effectiveness (OEE) to players in time sequences. We proposed a method called "Moving Coalition Analysis" (MCA) to observe performance trends of a coalition over time by treating each year or time as a player. In this method, we divide a coalition into several subcoalitions and calculate the characteristic function of all subcoalitions. Then, we examine the collaboration of Performance Rate, Quality Rate, and Availability for each subcoalition using the Shapley value. This method shows the relationship between each player and its contributors and can show trends of an activity in a time sequence even though the value of OEE is constant. This concept is applicable to various sectors of maintenance management and can play a role in considering the balance of combinations of criteria when performing maintenance.
\end{abstract}

\section{Introduction}

An organization consists of several units, sections, or departments that all aim to achieve one goal. However, the contribution of each sector has different weighting factors. Therefore it is necessary to link every sector through communication and cooperation. For example, the contributions of volumes of sales are comprised from the output of a marketing department. From a marketing department perspective, results are oriented from several sets of contributions such as business hours, people, and funds. Therefore these conditions should be carefully taken into considerations during evaluation.

In the current manufacturing industry, maintenance is an indispensable factor that influences the production cost. According to a study by Mobley, between $15 \%$ to $40 \%$ (with an average of $28 \%$ ) of the total production cost is caused by the maintenance activity [1]. In Finland, maintenance costs account for about $5.5 \%$ of the gross sales of a company [2]. Typically, expenses related to maintenance increase with the advancement in equipment technology. Therefore, analysis and evaluation are necessary for maintenance.
The Overall Equipment Effectiveness (OEE) index is often used to monitor maintenance management. OEE is calculated from Performance Rate, Quality Rate, and Availability. We could say that Performance Rate, Quality Rate, and Availability are working together to achieve an OEE and their combinations in each period are always different. We define the elements that are working together and that have their own contribution to achieve a common goal in a certain period as a contributor and a period as a player.

From our survey, we found that Performance Rate, Quality Rate, and Availability are handled by different sections. For example, the Production department is responsible for Performance Rate. The Quality department and Maintenance department are responsible for Quality rate and Availability, respectively. Knowing the contribution factors of each distribution helps with ease of managing activities. The best tool to evaluate the contribution of each player is the Shapley value, proposed by Lloyd Shapley in 1953. It is a Game theory concept used to determine the fairest allocation of collectively gained profits between the several collaborative players [3]. 
Examples of application of the Shapley value are given as follows. Reyes uses the Shapley value as an approach for solving the transshipment problem for maintaining the state of the logistics network which consists of the manufacturer, wholesaler, and retailer [4]. Cooperation of these three players was computed and the state where the logistics network was stabilized was proposed. Petrosjan and Zaccour study the problem of allocation over time of total cost incurred by countries in a cooperation game of pollution reduction by calculating characteristic functions of all the possible coalitions, and apply the Shapley value to determine a fair distribution of the total cooperative cost among countries [5].

Previously performed maintenance activities continue to have an influential until today. Therefore, the contribution of each player from time to time should be evaluated for better performance monitoring. For this purpose, we proposed a method called "Moving Coalition Analysis" (MCA) where each year or time is treated as a player.

This paper evaluates the contribution of Performance Rate, Quality Rate, and Availability of OEE using the Moving Coalition Analysis. Section 2 describes the general model of Moving Coalition Analysis. In Section 3, an illustrative example of MCA is illustrated. Section 4 introduces the application of MCA for bakery production line as a case study. We offer a conclusion in Section 5.

\section{Moving Coalition Analysis}

Assume that there are $n$ players with $m$ contributor and let $w$ be the weight to the contributor. Any subset $S$ of the player set $N=(1, \ldots, n)$ is called a coalition. The record for the coalition $S$ is defined by

$$
x_{i}(S)=\sum_{j \in S} x_{i j} \quad(i=1, \ldots, m)
$$

where $x_{i j}$ is the record of player $j$ to the contributor $i$.

This coalition aims at obtaining the maximal outcome $c(S)$ :

$$
\begin{aligned}
c(S)= & \max \sum_{i=1}^{m} w_{i} x_{i}(S) \\
& \text { s.t. } \sum_{i=1}^{m} w_{i}=1, \quad w_{i} \geq 0(\forall i) .
\end{aligned}
$$

The $c(S)$, with $c(\varnothing)=0$, defines a characteristic function of the coalition $S$. Thus, we have a game in coalition form with transferable utility, as represented by $(N, c)[6]$.

The Shapley value of the game $(N, c)$ for the player $k$ is the average of its marginal contribution to all possible coalitions:

$$
\varphi_{k}(c)=\sum_{\text {all } S} \gamma_{n}(S)[c(S)-c(S-\{k\})]
$$

with weights of probability to enter into a coalition $S$ defined as following:

$$
\gamma_{n}(S)=\frac{(s-1) !(n-s) !}{n !}
$$

In (3) and (4), $n$ is the total number of all the participants, $s$ is the number of members in the Sth coalition, and $c(\cdot)$ is the characteristic function used for estimation of utility for each coalition. If a subset $S(\subset N)$ includes player $k, k$ 's marginal contribution is obtained as $c(S)-c(S-\{k\})[7,8]$.

Moving Coalition Analysis is a method to observe performance trends of a coalition over time by using the Shapley value to calculate the marginal contribution of each period in a sub-coalition formed by several periods or players. A coalition is devided into several sub-coalitions where each sub-coalition should consist of three or more players. A sub-coalition dropped one of its members and picked up a new member to form the next sub-coalition.

Let a coalition consist of $N$ players with $M$ contributors and let a sub-coalition consist of $k$ players. $(K \geq 3)$. Then, we will have $S=(N-K+1)$ sub-coalitions. For example, let $N=5$ and $K=3$, then the number of sub-coalition $S$ is 3 .

Assume that $\varphi_{s n}$ is the Shapley value in a sub-coalition. We then benchmark the Shapley Value obtained from each sub-coalition against the best value of each sub-coalition.

$$
\theta_{s n}=\frac{\varphi_{s n}}{(\text { best Shapley value of sub-coalitions) }} .
$$

For example, let $\varphi_{11}, \varphi_{12}, \varphi_{13}, \ldots, \varphi_{1 k}$ are the Shapley value in a sub-coalition and $\varphi_{12}$ is the best Shapley value in this sub-coalition. Then, we divided each $\varphi_{11}, \varphi_{12}, \varphi_{13}, \ldots, \varphi_{1 k}$ by $\varphi_{12}$ and obtained, $\theta_{11}, \theta_{12}, \theta_{13}, \ldots, \theta_{1 k}$ shown in Table 2 . We called this value as "Scale of Balance (SoB)". The allocation for each player is derived from combinations of player's contributions. High contribution with a good balance of combinations will lead to higher allocation to the player. We defined this phenomenon as $\mathrm{SoB}=1$.

$\theta_{s n}$ is SoB of sub-coalition no. $s$ in period $n$. For example, $\theta_{11}$ is the SoB of 1 st sub-coalition in period $t_{1}$ while the average of $S o B$ of 1 st sub-coalition is denoted by $\theta_{1}$. Change of the average of SoB shows the tendency of timely change of the activities.

\section{Moving Coalition Analysis for Collaboration Evaluation in Overall Equipment Effectiveness: An Illustrative Example}

In this section, we apply Moving Coalition Analysis to evaluate the collaboration of Performance Rate, Quality Rate, and Availability in Overall Equipment Effectiveness.

Assume that activities conducted in six periods produced data as shown in Table 3. OEE was derived as follows

$$
\begin{aligned}
\text { OEE }= & \text { Performance Rate }(\mathrm{PR}) \times \text { Quality Rate }(\mathrm{QR}) \\
& \times \text { Availability }(\mathrm{A})
\end{aligned}
$$

We use the same value of OEE but with different values of Performance Rate, Quality Rate, and Availability to strengthen the influence of these contributors.

In this example, the number of players $N=6$, and the number of players in each sub-coalition $K=3$. Therefore, the number of sub-coalitions $S=4$. Each sub-coalition is shown in Table 4(a) to Table 4(d). 
TABLE 1: The Shapley value.

\begin{tabular}{|c|c|c|c|c|c|c|c|c|c|c|c|c|}
\hline \multirow{2}{*}{ Contributor } & \multicolumn{12}{|c|}{ Player } \\
\hline & $t_{1}$ & $t_{2}$ & $t_{3}$ & $\cdots$ & $t_{k}$ & $t_{k+1}$ & $\cdots$ & $t_{s}$ & $t_{s+1}$ & $\cdots$ & $t_{N-1}$ & $t_{N}$ \\
\hline 1st sub-coalition & $\varphi_{11}$ & $\varphi_{12}$ & $\varphi_{13}$ & $\cdots$ & $\varphi_{1 k}$ & & & & & & & \\
\hline 2nd sub-coalition & & $\varphi_{22}$ & $\varphi_{23}$ & $\cdots$ & $\varphi_{2 k}$ & $\varphi_{2 k+1}$ & & & & & & \\
\hline$\vdots$ & & & & & & & & & & & & \\
\hline $\begin{array}{l}\text { sth sub-coalition } \\
\vdots\end{array}$ & & & & & & & & $\varphi_{s s}$ & $\varphi_{s s+1}$ & $\cdots \varphi_{s s+k}$ & & \\
\hline Sth sub-coalition & & & & & & & & & & $\varphi_{S N-k+1} \cdots$ & $\varphi_{S N-1}$ & $\varphi_{\mathrm{SN}}$ \\
\hline
\end{tabular}

Table 2: Assessment for Table 1.

\begin{tabular}{|c|c|c|c|c|c|c|c|c|c|c|c|c|c|}
\hline \multirow{2}{*}{ Contributor } & \multicolumn{13}{|c|}{ Player } \\
\hline & $t_{1}$ & $t_{2}$ & $t_{3}$ & $\cdots$ & $t_{k}$ & $t_{k+1}$ & $\cdots$ & $t_{s}$ & $t_{s+1}$ & $\cdots$ & $t_{N-1}$ & $t_{N}$ & Average SoB \\
\hline 1st sub-coalition & $\theta_{11}$ & $\theta_{12}$ & $\theta_{13}$ & $\cdots$ & $\theta_{1 \mathrm{k}}$ & & & & & & & & $\theta_{1}$ \\
\hline 2nd sub-coalition & & $\theta_{22}$ & $\theta_{23}$ & $\cdots$ & $\theta_{2 \mathrm{k}}$ & $\theta_{2 \mathrm{k}+1}$ & & & & & & & $\theta_{2}$ \\
\hline sth sub-coalition & & & & & & & & $\theta_{\mathrm{ss}}$ & $\theta_{\mathrm{ss}+1}$ & $\cdots \theta_{s s+k}$ & & & $\theta_{s}$ \\
\hline Sth sub-coalition & & & & & & & & & & $\theta_{\mathrm{SN}-k+1} \cdots$ & $\theta_{\mathrm{SN}-1}$ & $\theta_{\mathrm{SN}}$ & $\theta_{s}$ \\
\hline
\end{tabular}

From Table 4(a), we divided each row by row-sum to normalize the sum to 1 as shown in Table 5 .

From Table 5, maximum outcome of $t_{1}$ is given by

$$
c\left(t_{1}\right)=\max 0.3438 w_{1}+0.3344 w_{2}+0.3218 w_{3}
$$

subject to

$$
\begin{gathered}
w_{1}+w_{2}+w_{3}=1, \\
\mathrm{w}_{1}, \mathrm{w}_{2}, \mathrm{w}_{3} \geq 0,
\end{gathered}
$$

where $w$ is the weight of the contributor

The optimal solution, $c\left(t_{1}\right)=0.3438$ is obtained when $w_{1}=1, w_{2}=0$, and $w_{3}=0$. The underline in Table 5 indicates the maximum outcome of each column.

We enumerate all coalition's values for each contributor in Table 5. For example, the value of coalition $\left\{t_{1}, t_{2}\right\}$ for Performance Rate is given as $0.3438+0.3192=0.6630$. Coalitions $\left\{t_{1}, t_{3}\right\}$ and $\left\{t_{2}, t_{3}\right\}$ are calculated by $0.3438+$ $0.3371=0.6809,0.3192+0.3371=0.6563$, respectively. The maximum outcomes or characteristic functions for each column are underlined as shown in Table 6.

Player $t_{1}, t_{2}$, and $t_{3}$ created six different "ordering" or permutation as per Table 7 . In "ordering" $t_{1} t_{2} t_{3}$, player $t_{1}$ is the first comer to the coalition, followed by player $t_{2}$, and finally player $t_{3}$. Thus, from Tables 4 and 5 , marginal contribution of each player to coalition can be evaluated as below.

$t_{3}$ 's marginal contribution is

$$
c\left(\left\{t_{1}, t_{2}, t_{3}\right\}\right)-c\left(\left\{t_{1}, t_{2}\right\}\right)=1-0.6688=0.3312
$$

$t_{2}$ 's marginal contribution is

$$
c\left(\left\{t_{1}, t_{2}\right\}\right)-c\left(\left\{t_{1}\right\}\right)=0.6688-0.3438=0.3250 .
$$

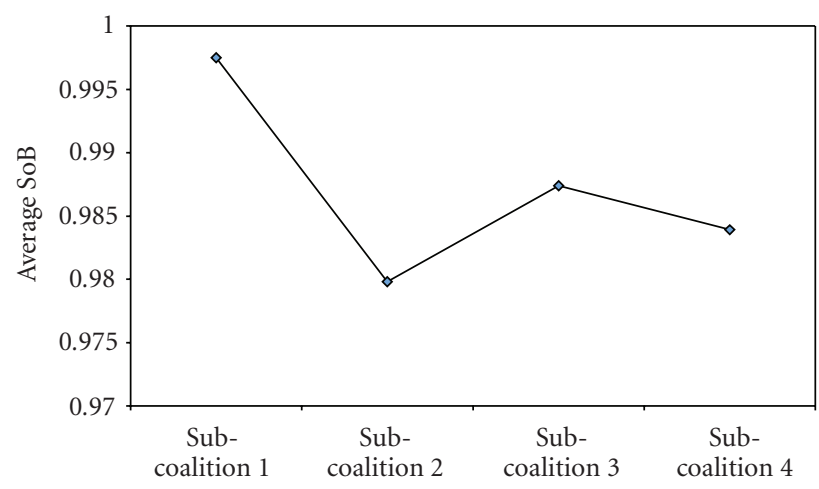

Figure 1: Trend of timely change.

Lastly, $t_{1}$ 's marginal contribution is

$$
c\left(\left\{t_{1}\right\}\right)=0.3438
$$

The same calculation then was repeated for the other "ordering" form. The results are shown in Table 7. Shapley value, as shown in Table 8 is the average of the marginal contributions of each player. In Table 8, the best Shapley value among players is $t_{3}$. Then, we normalized the best Shapley value to 1 to obtain the score for other players against the best Shapley value. As a result, we can say that $t_{1}$ and $t_{2}$ are $99.60 \%$ and $99.63 \%$ of $t_{3}$, respectively.

This process is repeated for the second, third, and fourth sub-coalition. Then, we calculated the average SoB for each sub-coalition as shown in Table 9. The graph is plotted in Figure 1. 
TABle 3: Example of a Coalition.

\begin{tabular}{lcccccc}
\hline \multirow{2}{*}{ Contributor } & \multicolumn{5}{c}{ Player } & \multicolumn{1}{c}{$t_{5}$} \\
\hline PR & $t_{1}$ & $t_{2}$ & $t_{3}$ & $t_{4}$ & 90.87 & 90.87 \\
OR & 90.86 & 84.35 & 89.08 & 78.02 & 97.83 \\
A & 90.86 & 90.86 & 89.97 & 97.86 & 79.27 & 87.56 \\
OEE & 90.86 & 97.87 & 93.59 & 98.25 & 95.84 & 0.7501 \\
\hline
\end{tabular}

Table 4: Sub-Coalition.

(a) 1st sub-coalition

\begin{tabular}{lcccc}
\hline & $t_{1}$ & $t_{2}$ & $t_{3}$ & sum \\
\hline PR & 90.86 & 84.35 & 89.08 & 264.29 \\
QR & 90.86 & 90.86 & 89.97 & 271.69 \\
A & 90.86 & 97.87 & 93.59 & 282.32 \\
\hline
\end{tabular}

(b) 2nd sub-coalition

\begin{tabular}{lcccc}
\hline & $t_{2}$ & $t_{3}$ & $t_{4}$ & sum \\
\hline PR & 84.35 & 89.08 & 78.02 & 251.45 \\
QR & 90.86 & 89.97 & 97.86 & 278.69 \\
A & 97.87 & 93.59 & 98.25 & 289.71 \\
\hline
\end{tabular}

(c) 3rd sub-coalition

\begin{tabular}{lcccc}
\hline & $t_{3}$ & $t_{4}$ & $t_{5}$ & sum \\
\hline PR & 89.08 & 78.02 & 98.73 & 265.83 \\
QR & 89.97 & 97.86 & 79.27 & 267.10 \\
A & 93.59 & 98.25 & 95.84 & 287.68 \\
\hline
\end{tabular}

(d) 4th sub-coalition

\begin{tabular}{lcccc}
\hline & $t_{4}$ & $t_{5}$ & $t_{6}$ & sum \\
\hline PR & 78.02 & 98.73 & 90.87 & 267.62 \\
QR & 97.86 & 79.27 & 94.28 & 271.41 \\
A & 98.25 & 95.84 & 87.56 & 281.65 \\
\hline
\end{tabular}

TABLE 5: Normalized values for 1st sub-coalition.

\begin{tabular}{lcccc}
\hline & $t_{1}$ & $t_{2}$ & $t_{3}$ & sum \\
\hline PR & $\underline{0.3438}$ & 0.3192 & $\underline{0.3371}$ & 1 \\
QR & 0.3344 & 0.3344 & 0.3311 & 1 \\
A & 0.3218 & $\underline{0.3467}$ & 0.3315 & 1 \\
\hline
\end{tabular}

TABLE 6: Coalition and characteristic function for 1st sub-coalition.

\begin{tabular}{llll}
\hline Contributor & Coalition & \\
& $\left\{t_{1}, t_{2}\right\}$ & $\left\{t_{1}, t_{3}\right\}$ & $\left\{t_{2}, t_{3}\right\}$ \\
\hline PR & 0.6630 & $\underline{0.6809}$ & 0.6563 \\
QR & $\underline{0.6688}$ & 0.6655 & 0.6655 \\
A & 0.6685 & 0.6533 & 0.6782 \\
\hline
\end{tabular}

TABLE 7: Marginal contribution for 1st sub-coalition.

\begin{tabular}{lccc}
\hline Ordering & \multicolumn{3}{c}{ Player } \\
$t_{1}$ & $t_{2}$ & $t_{3}$ \\
\hline$t_{1} t_{2} t_{3}$ & 0.3438 & 0.3250 & 0.3312 \\
$t_{1} t_{3} t_{2}$ & 0.3438 & 0.3191 & 0.3371 \\
$t_{2} t_{1} t_{3}$ & 0.3221 & 0.3467 & 0.3312 \\
$t_{2} t_{3} t_{1}$ & 0.3218 & 0.3467 & 0.3315 \\
$t_{3} t_{1} t_{2}$ & 0.3438 & 0.3191 & 0.3371 \\
$t_{3} t_{2} t_{1}$ & 0.3218 & 0.3411 & 0.3371 \\
\hline
\end{tabular}

TABLE 8: The Shapley value and SoB for 1st sub-coalition.

\begin{tabular}{lccc}
\hline & $t_{1}$ & $t_{2}$ & $t_{3}$ \\
\hline Shapley value & 0.33285 & 0.33295 & 0.3420 \\
SoB & 0.9960 & 0.9963 & 1.0 \\
\hline
\end{tabular}

TABle 9: Average SoB.

\begin{tabular}{lccccccc}
\hline & $t_{1}$ & $t_{2}$ & $t_{3}$ & $t_{4}$ & $t_{5}$ & $t_{6}$ & $\begin{array}{l}\text { Average } \\
\text { SoB }\end{array}$ \\
\hline $\begin{array}{l}\text { 1st sub- } \\
\text { coalition }\end{array}$ & 0.9960 & 0.9963 & 1.0000 & & & & 0.9974 \\
$\begin{array}{l}\text { 2nd sub- } \\
\text { coalition }\end{array}$ & 0.9630 & 1.0000 & 0.9765 & & & 0.9798 \\
$\begin{array}{l}\text { 3rd sub- } \\
\text { coalition }\end{array}$ & & & 0.9742 & 0.9879 & 1.0000 & & 0.9874 \\
$\begin{array}{l}\text { 4th sub- } \\
\text { coalition }\end{array}$ & & & & 0.9767 & 0.9749 & 1.0000 & 0.9839 \\
\hline
\end{tabular}

\section{A Case Study}

As a case study, the Overall Equipment Effectiveness and its contributor for five periods of a bakery production line are shown in Table 10 [9]. Let period 1, period 2, period 3, period 4 , and period 5 be $t_{1}, t_{2}, t_{3}, t_{4}$, and $t_{5}$, respectively. In this case, the number of players $N=5$, and the number of players in each sub-coalition $K=3$. Therefore, the number of sub-coalitions $S=3$. Each sub-coalition is shown in Table 11(a) through Table 11(c).

The SoB can be calculated by the same method as described in the earlier exercise.. The acquired result from this exercise is shown in Table 12. 
TABLE 10: OEE and its contributor for a bakery production line.

\begin{tabular}{lccccc}
\hline Contributor & \multicolumn{3}{c}{ Player } & $t_{4}$ & $t_{5}$ \\
\hline A & $t_{1}$ & $t_{2}$ & $t_{3}$ & 91.62 & 90.21 \\
QR & 88.96 & 89.46 & 91.09 & 93.26 & 97.18 \\
PR & 87.41 & 89.31 & 91.84 & 88.70 & 90.77 \\
OEE & 80.21 & 84.39 & 97.03 & 0.7579 & 0.7957 \\
\hline
\end{tabular}

(source: Tsarouhas, 2007 [9]).

Table 11: Sub-Coalition for Table 10.

(a) 1st sub-coalition

\begin{tabular}{lcccc}
\hline & $t_{1}$ & $t_{2}$ & $t_{3}$ & sum \\
\hline A & 88.96 & 89.46 & 91.09 & 269.51 \\
QR & 87.41 & 89.31 & 91.84 & 268.56 \\
PR & 80.21 & 84.39 & 97.03 & 261.63 \\
\hline
\end{tabular}

(b) 2nd sub-coalition

\begin{tabular}{lcccc}
\hline & $t_{2}$ & $t_{3}$ & $t_{4}$ & sum \\
\hline A & 89.46 & 91.09 & 91.62 & 272.17 \\
QR & 89.31 & 91.84 & 93.26 & 274.41 \\
PR & 84.39 & 97.03 & 88.70 & 270.12 \\
\hline
\end{tabular}

(c) 3rd sub-coalition

\begin{tabular}{lcccc}
\hline & $t_{3}$ & $t_{4}$ & $t_{5}$ & sum \\
\hline A & 91.09 & 91.62 & 90.21 & 272.92 \\
QR & 91.84 & 93.26 & 97.18 & 282.28 \\
PR & 97.03 & 88.70 & 90.77 & 276.50 \\
\hline
\end{tabular}

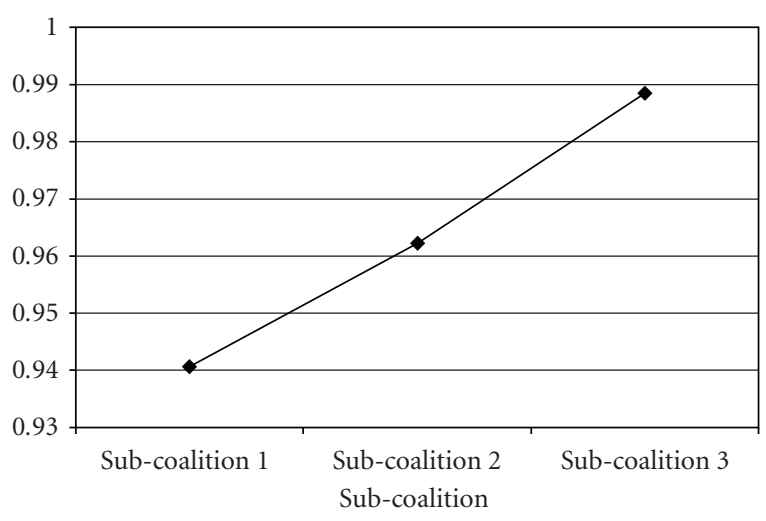

Figure 2: Trend of timely change for Table 10.

From Table 12, authors plotted the graph as shown in Figure 2 to indicate the trend of timely change for the data in Table 10.

\section{Discussion and Conclusion}

From Figure 1, the changes of graph can be seen even though the value of OEE is constant. These changes were created due to the different values of marginal contributions of each player. These changes show which activity is most excellent in performance. From Figure 1, we learned that coalitions $\left\{t_{1}, t_{2}, t_{3}\right\}$ have a better average $\mathrm{SoB}$ in comparison to coalitions $\left\{t_{2}, t_{3}, t_{4}\right\}$. Since $t_{2}$ and $t_{3}$ are the common players in these two coalitions, the slope shows the differential of $t_{1}$ and $t_{4}$. From Table 4 , we noticed that $t_{1}$ has a better balanced combination over $t_{4}$ where all contributors in $t_{1}$ have the same value. This information is important because we do not want any section or department in the same organization to be left behind. The section or department that leads ahead can share their knowledge or experience to another sections that are left behind.

Table 12 indicates that $t_{3}$ has a good balance of activity with a high contribution of $\mathrm{PR}, \mathrm{QR}$, and $\mathrm{A}$, followed by $t_{5}$ while the value of the average $\mathrm{SoB}$ for sub-coalition 3 was the highest in comparison with the others. This information is useful to assist managers in estimating the target for the following periods. Considering the current condition for this production line, the values of $\mathrm{PR}, \mathrm{QR}$, and A should be around 91 to 97 . Adding additional resources such as man power and budget might increase OEE. However, this will also lead to increases in the production cost. Therefore, the best way to estimate the target is to set the target using current resources by allocating these resources from a higher contributor to a lower contributor in a good balance. MCA will help to check and measure the combinations balance for each contributor.

The example in Table 9 shows that sub-coalition 1 with $t_{1}$, one of the members has the same value of $P R, Q R$, and $A$ recorded a high average $\mathrm{SoB}$ in comparison with other sub-coalitions while the value of OEE for each member is the same. We believe that higher OEE alone is not enough to ensure the effectiveness of the maintenance activities but must be followed by a good balance of the combination of PR, QR, and A.

This method can exhibit the relationship between each player and contributor and can play a role in considering the balance of combination between contributors when performing the activity. Therefore, an activity's precise change can be observed by using this method.

New viewpoints can also be estimated with the combination of other methods. For example, the combination of Data Envelopment Analysis (DEA) and the Shapley value was proposed by Nakabayashi and Tone in their "Egoist's dilemma" theory [6]. Wu et al. treated the decision making unit (DMU) of DEA which is used as a player of a cooperative game, and computed the characteristic function value of each coalitions. Finally, using Shapley value, the efficiency of each DMU is computed [10]. 
TABle 12: Average SoB for Table 11.

\begin{tabular}{lcccccc}
\hline & $t_{1}$ & $t_{2}$ & $t_{3}$ & $t_{4}$ & $t_{5}$ & Average SoB \\
\hline 1st sub-coalition & 0.8979 & 0.9239 & 1.0000 & & 0.9406 \\
2nd sub-coalition & & 0.9238 & 1.0000 & 0.9630 & 0.9623 \\
3rd sub-coalition & & & 1.0000 & 0.9709 & 0.9947 & 0.9885 \\
\hline
\end{tabular}

We cannot ignore the fact that cost is one of the important criteria in performing maintance. However, MCA, as well as OEE is output-oriented and doest not involve any input criteria such as cost or man power in its calculations. MCA is a method to evaluate the result of the activities. In our opinion, the best way to involve cost in evaluating maintenance is to use DEA where DEA is the method to evaluate the progress/process of the activities, in our case, the maintenance activities. Our future work is to propose an evaluation technique that combines the MCA and DEA which can cater to both input criteria and output criteria.

\section{References}

[1] R. K. Mobley, An Introduction to Predictive Maintenance, Van Nostrand Reinhold, New York, NY, USA, 1990.

[2] K. Komonen, "A cost model of industrial maintenance for profitability analysis and benchmarking," International Journal of Production Economics, vol. 79, no. 1, pp. 15-31, 2002.

[3] E. A. Roth, The Shapley Value, Cambridge University Press, New York, NY, USA, 1988.

[4] P. M. Reyes, "Logistics networks: a game theory application for solving the transshipment problem," Applied Mathematics and Computation, vol. 168, no. 2, pp. 1419-1431, 2005.

[5] L. Petrosjan and G. Zaccour, "Time-consistent Shapley value allocation of pollution cost reduction," Journal of Economic Dynamics and Control, vol. 27, no. 3, pp. 381-398, 2003.

[6] K. Nakabayashi and K. Tone, "Egoist's dilemma: aDEA game," Omega, vol. 34, no. 2, pp. 135-148, 2006.

[7] W. W. Cooper, L. M. Seiford, and K. Tone, Data Envelopment Analysis: A Comprehensive Text with Models, Applications, References and DEA-Solver Software, Springer, London, UK, 2nd edition, 2006.

[8] M. Conklin, K. Powaga, and S. Lipovetsky, "Customer satisfaction analysis: identification of key drivers," European Journal of Operational Research, vol. 154, no. 3, pp. 819-827, 2004.

[9] P. Tsarouhas, "Implementation of total productive maintenance in food industry: a case study," Journal of Quality in Maintenance Engineering, vol. 13, no. 1, pp. 5-18, 2007.

[10] J. Wu, L. Liang, and F. Yang, "Determination of the weights for the ultimate cross efficiency using shapley value in cooperative game," Expert Systems with Applications, vol. 36, no. 1, pp. 872-876, 2009. 